\title{
Bayesian geo-additive spatial modelling of HIV prevalence using data from population-based surveys
}

\author{
Samson B. Adebayo ${ }^{1}$, Ezra Gayawan², Adeniyi F. Fagbamigbe ${ }^{3}$, Fatai W. Bello ${ }^{4}$ \\ ${ }^{1}$ National Agency for Food and Drug Administration and Control, Abuja, Nigeria \\ ${ }^{2}$ Department of Statistics, Federal University of Technology, Akure, Nigeria \\ ${ }^{3}$ Department of Epidemiology and Medical Statistics, College of Medicine, University of Ibadan, Nigeria \\ ${ }^{4}$ Department of Health Planning and Management, University of Ilorin, Ilorin, Nigeria
}

\begin{abstract}
Introduction: Estimates of human immunodeficiency virus (HIV) prevalence in Nigeria have been based on the data from HIV surveillance and sentinel studies among pregnant women attending antenatal clinics at some selected sentinel sites. However, such data overestimate HIV prevalence. This paper explores possible geographical variations in HIV prevalence among the general population of males and females based on two waves of the National HIV/acquired immune deficiency syndrome (AIDS) and Reproductive Health Surveys.

Material and methods: Data were extracted from the cross-sectional 2007 and 2012 National HIV/ AIDS and Reproductive Health Serological Surveys of men (15-64 years) and women (15-49 years) covering all states of Nigeria. Bayesian geo-additive modelling technique was employed for analysis. Appropriate prior distributions were assigned to the different types of variables in the models and inference was based on the Markov Chain Monte Carlo (MCMC) technique. Models of different specifications were considered.

Results: The findings reveal significant spatial variations at a highly disaggregated level of states in Nigeria. The nonlinear effects of respondents' age show a similar pattern of HIV prevalence for male, female and the combined respondents, implying that HIV prevalence is peak among middle-age individuals, from where it declines with age. Also, the results reveal a downward change in HIV prevalence in Nigeria between 2007 and 2012.

Conclusions: When these findings are taken into consideration in designing intervention strategies, it is believed that each state can be targeted with the right intervention(s). This can also lead to efficient utilization of the scarce resources witnessed globally and more importantly with the economic recession in Nigeria.
\end{abstract}

HIV AIDS Rev 2019; 18, 4: 247-260 DOI: https://doi.org/10.5114/hivar.2019.83852

Key words: Markov chain Monte Carlo, Nigeria, spatial distribution, HIV/AIDS, Bayesian analysis.

Address for correspondence: Samson B. Adebayo, National Agency for Food and Drug Administration and Control, Plot 2032 Olusegun Obasanjo Way, 900009, Abuja, Nigeria, phone: +2347033979837, e-mail: adebayosam@hotmail.com
Article history:

Received: 30.04 .2018

Received in revised form: 02.10 .2018

Accepted: 19.11.2018

Available online: 01.03.2019
International Journal of HIV-Related Problems

HIV \& AIDS

R e v i e w 


\section{Introduction}

Designing appropriate strategies to mitigate the scourge of human immunodeficiency virus (HIV) epidemics requires an adequate knowledge of the correlates and the important risk factors. A proper understanding of the spatial components of the epidemics at smaller geographical units in any country could play a critical role in structuring, developing and implementing appropriate strategies that will be impactful on the people at the localized settings [1]. To this end, reliable data that are made available on the spread of HIV and its risk factors in any population when available at smaller geographical units can be vital for any effective response to the epidemic and its consequences. In countries with generalized epidemics, national estimates of HIV prevalence and trends in the adult population at the national and sub-national levels are generally derived indirectly from HIV surveillance among pregnant women attending selected antenatal clinics [2-4].

People around the same geographical areas often share similar beliefs and culture, which often dictate their common exposure to diseases including HIV. Therefore, countries with multiple and diverse cultures are likely to record huge variations in the prevalence of HIV based on their geographic locations. It is frequently assumed that affluent countries have escaped the strongest influence of the HIV virus because they enjoy better health care and educational systems that reduce the spread of the epidemics. By contrast, most developing countries especially in the sub-Saharan Africa have continued to battle with the epidemic notwithstanding that the basic history of the HIV virus reveals that the first crisis of HIV and the disease it causes, acquired immune deficiency syndrome (AIDS), occurred in the USA (one of the richest countries in the world) in the 1980s [5]. Globally, nearly half (35.4 million) of the over 77.3 million people infected with HIV from the beginning of the epidemic until the end of 2017 lost their lives due to HIV/AIDSrelated infections [6]. At the close of 2017, almost 37 million persons were living with HIV globally [6]. Undoubtedly, Africa disproportionately bears the burden of HIV compared to other continents, with $4.1 \%$ prevalence, and is home to 7 in every 10 persons living with HIV/AIDS globally [6-8]. A huge disparity in the HIV burden across African countries however exists. A recent UNAIDS report shows that Nigeria, Uganda and South Africa jointly share half of the yearly new infections in sub-Saharan Africa while Nigeria alone was responsible for $60 \%$ of new HIV infections in Western and Central Africa in 2015 [9].

Today Sub-Saharan Africa is in the midst of a HIV/AIDS crisis, and with it is the stigma that AIDS is a virus of poverty. The ActionAid states that "the HIV/AIDS crisis in developing countries is inextricably linked with poverty" [10]. Once seen as being a 'gay' virus, but now called a 'black' virus, HIV/AIDS is obviously associated with poverty and suffering, something previously acquainted with stereotypes of famine in Ethiopia [11].

Among the neighbouring West African countries, Nigeria was rated as the third most affected country, behind only Cameroon (5.3\%) and Gabon (5.2\%) (USAID, 2012). The latest national population-based Nigeria serological survey shows the HIV prevalence to be $3.4 \%$ [12] compared with 19.2\% in South Africa and 12.9\% in Zambia [9]. Although Nigeria's figure seems low at 34 per 1,000 persons being HIV positive, if one takes into account that at least 180 million people currently live in the country, it would translate to about 4 million Nigerian inhabitants infected with HIV [13]. This has placed Nigeria as the country with the second largest HIV epidemic globally [14]. Although there is evidence of reduction in the yearly cases of new infections from about 317,000 in 2003 to 240,000 in 2013, a colossal total of 175,000 died from HIV/AIDS related cases in 2014 alone in the country, partly because only $45 \%$ of the over 1.6 million persons needing antiretroviral therapy (ART) were treated in that year [14]. Nearly 2 million new HIV infections among adults were recorded in 2015 globally and about a third of people living with HIV are virally suppressed [9]. In most of the developing countries mostly affected, condom use remains low among the most at-risk groups, and the majority of youths lack adequate knowledge of HIV and its mode of transmission [9]. In sub-Saharan Africa, it is estimated that the yearly gap in male condom use is in excess of 3 billion [9]. At least one in every four persons residing in Nigeria reportedly knows at least one person who either contracted HIV or died of AIDS [15, 16], and therefore, a multi-sectoral approach is required to combat the menace of HIV/AIDS.

As with many other health indicators, there are spatial variations in HIV prevalence in Nigeria. For instance, based on the rural-urban setting, the prevalence is about $4 \%$ in rural areas but $3 \%$ in urban areas. Considering the geographical zones, HIV prevalence in the South-South zone is estimated to be $5.5 \%$ compared with $1.8 \%$ in the neighbouring South East zone $[14,17]$. Bringing it down to state level, whereas the estimates are as high as $15.2 \%$ and $10.5 \%$ in Rivers and Tabara states respectively, Zamfara and Ekiti states have estimates of $0.4 \%$ and $0.2 \%$ respectively [15]. Interestingly, none of these states with the highest or lowest estimates share a common boundary [15]. The estimated prevalence also indicates the substantial variations based on demographic factors such as sex, marital status, age and sexual risk behaviours. The prevalence was higher among females (4.0\%) than males (3.2\%) [18]. The latest of the national surveys in 2012 showed a slightly lower general population HIV prevalence of $3.4 \%$ in Nigeria, ranging from $3.2 \%$ to $3.6 \%$ [15]. Findings from Adebayo et al. [19] and Fagbamigbe et al. [20] showed that HIV prevalence is about twice as high among women of reproductive age compared with their male counterparts. Furthermore, the prevalence among formerly married women (i.e. divorced, widowed and separated) is about three times that of their counterparts who were currently married or cohabiting with sexual partners. Expectedly, the prevalence among men and women who engaged in transactional sex is more than twice as high for those who had never engaged in transactional sex. 
Studies have demonstrated that in analysing health indicators, assuming a linear effect for metrical covariates is always too rigid and can result in misleading findings [21, 22]. HIV prevalence by age has been shown to have a nonlinear relationship, assuming an inverted U-shape [23]. In particular, HIV prevalence is low among people below the age of 18 but increases up to the age of 35-40 then starts declining with every unit increase in age [24]. There is no doubt in the fact that estimating the prevalence at smaller geographic units can provide information on locations with high and low risks to policymakers. These estimates, from survey reports, may, however, not be reliable because they depend entirely on the size of the sample taken from the particular location. On the other hand, model-based estimates result in a mechanism that enhances local estimation by combining data from nearby locations in the estimation process, thereby improving local estimates even in places with scanty data points. Analysing HIV/AIDS surveillance data from pregnant women attending antenatal care services in Nigeria, Eze [25] obtained a spatial trend that indicates low prevalence in the western and northern part of the country and moderate levels in the eastern and South-South zones. However, the present study intends to analyse the spatial distribution at more localized units taking the states that make up the federating units of the country as units of analysis based on data from the National HIV/AIDS Reproductive Survey involving male and female respondents from selected households, rather than only pregnant women presenting for antenatal care. In this paper, a Bayesian geo-statistical approach based on the Markov Chain Monte Carlo (MCMC) simulation technique was adopted.

\section{Material and methods}

The study relies on data from cross-sectional households surveys of men aged 15-64 years and women aged 15-49 years conducted in 2007 and 2012 as part of the National HIV/AIDS and Reproductive Health and Serological Survey (NARHS Plus) covering both rural and urban areas in the 36 states and the Federal Capital Territory (FCT) of Nigeria. The survey contained both behavioural and serological components.

For both surveys, eligible respondents were selected through multi-stage cluster sampling. At the first stage, rural and urban areas from each state and the FCT were selected, while the second stage involved the selection of 30 Enumeration Areas (EA) within each of the selected rural and urban areas. At the final stage, the households to participate in the surveys were selected. Pre-tested structured questionnaires consisting of questions adapted from the UNAIDS general population HIV/AIDS indicators and the Demographic and Health Survey questionnaires were administered to the female and male respondents. Enumerators, including HIV counsellors/testers who were versed in both English and local languages, were recruited for data collection. They were trained with the aim of acquainting them with the survey instruments and methodologies prior to the survey.

\section{HIV testing and validation}

A linked anonymous testing approach with the provision of test results was adopted in the survey. Comprehensive counselling was given to the respondents, after which their informed consent was obtained before testing could be conducted. The testing algorithm consisted of the collection of five blood spots from a finger prick on the same filter paper card; these were stored as dried blood spots (DBS). STAT-PAK and Determine rapid test kits were used for onthe-spot testing with the immediate provision of test results. All survey instruments including the DBS and the questionnaire were linked anonymously using a unique random identification number. For quality control purposes, all positive samples, all discordant samples from rapid testing in the field, and a randomly selected sample of $10 \%$ of all negative samples were re-tested at the central laboratory using ELISA and Western blot as the decider.

\section{Data}

Data analysed in the study are those of respondents who participated in the behavioural aspect of the survey and consented to HIV testing. A total of 11,521 and 31,235 potential respondents were identified for the survey in 2007 and 2012 respectively. Of these, a total of 9,031 comprising 4,195 females and 4,836 males; and 24,115 comprising 12,079 females and 12,036 males, consented and participated in the HIV counselling and testing during 2007 and 2012 surveys. The refusal rates for females and males during the 2007 survey were $21.8 \%$ and $21.5 \%$ respectively while the refusal rate was approximately $22.8 \%$ for both females and males during the 2012 survey. The HIV test refusal in Nigeria is, however, higher than the $10 \%$ reported previously [26]. All analyses involving HIV status in the present study were based on the 9,031 (2007) and 24,115 (2012) respondents with both behavioural survey results and valid HIV test results.

\section{Dependent variable}

The outcome/dependent variable of interest in this study was a binary outcome indicating the HIV sero-status test result obtained from the respondents who were interviewed and consented to HIV testing. A reactive HIV test was coded " 1 " and non-reactive outcomes as " 0 ". HIV prevalence was therefore computed as numbers of " 1 "s out of the total respondents interviewed and tested divided by addition of numbers of " 1 "s and "0"s.

\section{Independent variable}

Based on existing literature, the following socio-demographic characteristics were explored in this paper: marital status, location of residence, educational attainment, geopolitical zones, age, self-reported sexual behaviour within the 12 months preceding the survey, age at first sex, experience of sexually transmitted infections (STIs), multiple part- 
nering, transactional sex, i.e., sex in exchange for gifts/favours, comprehensive knowledge of HIV, and state (geographical locations) where respondents resided at the time of the survey. Marital status was grouped into 'never married', 'currently married/cohabiting', and 'formerly married' (i.e. separated, divorced, or widowed). The state of residence of the respondents was geo-referenced for spatial analysis. Nigeria consists of 36 states and a Federal Capital Territory, Abuja.

Operational definition: Prevalence "is the proportion of a particular population found to be affected by a medical condition (typically a disease) at a point in time". HIV prevalence is, therefore, the proportion of all HIV tested respondents who were found positive.

\section{Statistical model and data analysis}

\section{Model}

The spatial analysis is based on the concept of the structured additive regression model for a binary response. The response variable $\left(y_{i j k}, z_{k}, v_{k}, r_{j} s_{i}\right) y_{i j k}$ is the HIV serostatus of the $j^{\text {th }}$ person living in the $i^{\text {th }}$ district/state. The distribution of this response variable belongs to the exponential family with mean $\mu=E(y \mid z, \beta) f(y)=\theta^{y} e^{-y} \quad y>0$ which can be linked to a linear predictor $\eta$ thus:

$$
\mu=h(\eta) \quad \eta=z^{\prime} \beta
$$

where $h$ is a response function and $\beta$ a regression parameter. In the present case, available covariates include spatial, metrical and categorical, which can then be considered within the framework of the generalized additive model (27). Following the structured additive modelling, the predictor $\eta_{i j}$ can be defined as

$$
\eta_{i j}=\text { time }_{2}+\sum_{j=1}^{p} f_{i j}\left(z_{i j}\right)+f_{\text {spat }}\left(s_{i}\right)+v_{i j} \beta
$$

where $f$ is the nonlinear unknown smooth function of the covariate age $(z), f_{\text {spat }}$ is the nonlinear spatial effect $(s)$, which can be divided into structured and unstructured random effects, and $\beta$ is the regression coefficients for the categorical covariates $(v)$.

\section{Bayesian inference}

Parameter estimation follows a Bayesian procedure where the different parameters and functions in the proposed model of the observed data are assigned prior distributions. Independent diffuse priors are assumed on the parameters of categorical effects, which produce results similar to the classical estimation procedure. For the non-linear effects, a Bayesian P-splines prior based on Lang and Brezger [28] and Brezger and Lang [29] was assumed. This enables nonparametric estimation of the function $f$ as a linear combination of the basis function (B-splines)

$$
p(z)=\sum_{t=1}^{m} \alpha_{t} B_{t}(z)
$$

where $B_{t}(z)$ are B-splines and the coefficients $\alpha_{t}$ are further defined to follow first or second order Gaussian random walk smoothness priors.

A Gaussian Markov random field prior was assumed for the geographical effects $f_{\text {spat }}(s), s=1, \ldots, S$. Basically, this is an extension of first-order random walk priors to twodimensional spatial arrays; see Rue and Held [30] for general information.

To estimate the smoothing parameters for non-linear and spatial effects simultaneously, highly dispersed but proper hyper-priors are assigned to them. An inverse gamma distribution with hyperparameters $a$ and $b$ is chosen for the variance components, e.g. $\tau^{2} \sim \operatorname{IG}(a, b)$. Standard choices of hyperparameters are $a=1$ and $b=0.005$ or $a=b=0.001$. The basis of inference in this study is carried out through computationally efficient Markov Chain Monte Carlo (MCMC) techniques.

\section{Data analysis of the models}

In order to understand the influence of both observed covariates and spatial variations as well as time on HIV prevalence in Nigeria, several models of different specifications were explored and the deviance information criterion (DIC) [32] was adopted for model diagnostics where the model with the smallest DIC among a set of similar models was considered the best. Several models were explored to understand the behaviour of the mixing and convergence in an attempt at studying the sensitivity to the choice of priors. Six of the fitted models are defined as follows:

$$
\mathrm{M}_{1}: \eta=\text { time }_{2}+f_{\text {spat }}\left(s_{i}\right)
$$

$\mathrm{M}_{2}: \eta=$ time $_{2}+f_{\text {spat }}\left(s_{i}\right)+\mathrm{f}($ Age of respondents $)+$ geopolitical zones + bio-demographic characteristics

$\mathrm{M}_{3}: \eta=$ time $_{2}+f_{\text {spat }}\left(s_{i}\right)+\mathrm{f}($ Age of respondents $)+$ geopolitical zones + bio-demographic characteristics + sexual behaviour

$\mathrm{M}_{4}: \eta=$ time $_{2}+f_{\text {spat }}\left(s_{i}\right)+\mathrm{f}($ Age of respondents $)+$ geopolitical zones + bio-demographic characteristics + sexual behaviour + knowledge indicators

$\mathrm{M}_{5}: \eta=$ time $_{2}+\mathrm{f}($ Age of respondents $)+$ geopolitical zones + bio-demographic characteristics + sexual behaviour + knowledge indicators

$\mathrm{M}_{6}: \eta=$ time $_{2}+f_{\text {str }}\left(s_{i}\right)+f_{\text {unstr }}\left(s_{i}\right)+\mathrm{f}($ Age of respondents $)$ + bio-demographic characteristics + sexual behaviour + knowledge indicators

These models were run for all the respondents (combined for males and females), male only and female only data. In the presentation and discussion of results, we present models with no spatial component and those with structured and unstructured spatial components.

\section{Results}

Table 1 presents findings from the descriptive analysis showing changes in the prevalence of HIV between 2007 and 2012 


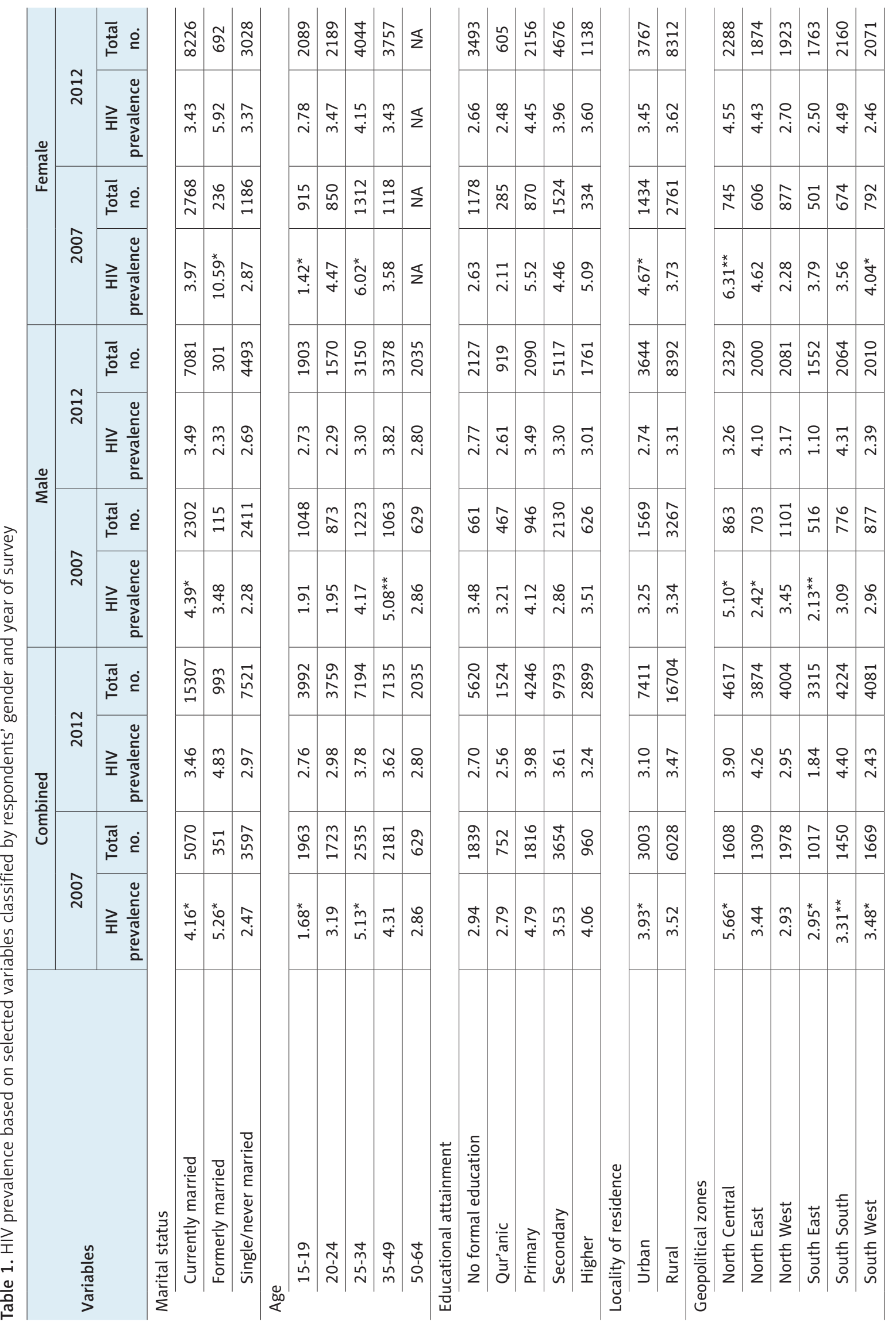




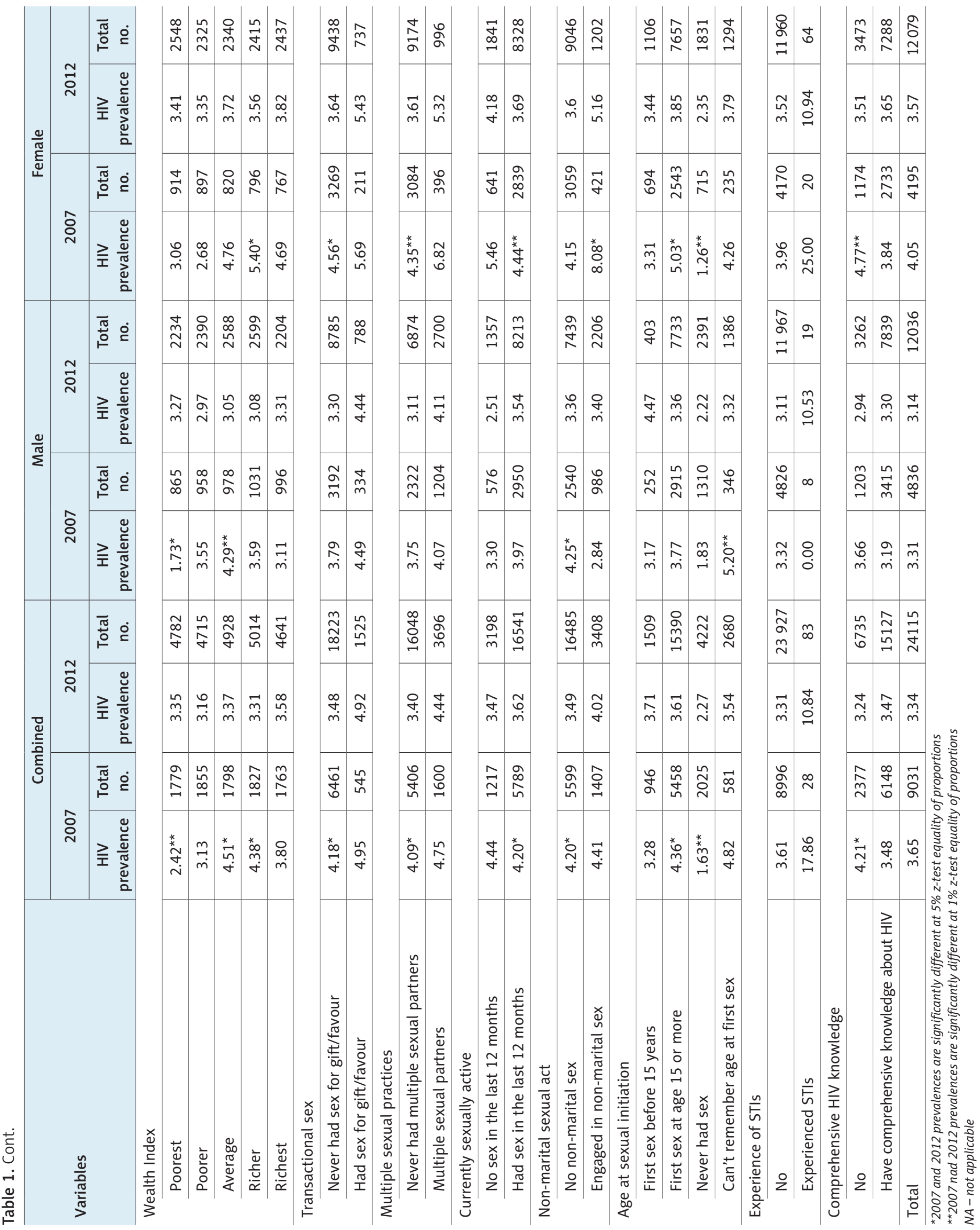


according to some selected characteristics. Generally, the findings show a significant decline in HIV prevalence in 2012 compared to 2007 among currently married and formally married subjects for both males and females as well as both combined.

Considering respondents' age, HIV prevalence significantly increases for respondents in the age group 15-19 years. Of concern is the prevalence that doubled in the same age group for females. A significant decline in HIV prevalence for the age group 25-34 years was evident for the combined and only female respondents, while for the male respondents, a significant decline was only evident in the age group 35-49 years. No significant association was evident between HIV prevalence and educational attainment. HIV prevalence among respondents who resided in urban areas witnessed a significant decline for combined respondents and females, but the decline was not significant for the males.

Findings shown in Table 1 further provide insightful information about significant spatial variations in HIV prevalence in Nigeria between 2007 and 2012 at the zonal level. While North Central, South East, and South West zones witnessed a significant decline in HIV prevalence between 2007 and 2012, the South-South zone witnessed a highly significant increase $(p<0.001)$ in HIV prevalence in the combined data.

Respondents in the poorest and poorer households showed an increase in HIV prevalence between 2007 and 2012. Only respondents in the average, richer and richest wealth quintiles experienced a decline during this period. A significant decline in HIV prevalence between the two time points was evident among respondents who never had sex in exchange for a gift/favour, had no non-marital sexual act, or never had multiple sexual partners in the last 12 months. Respondents who had sex in the last 12 months also witnessed a significant decline in HIV prevalence between 2007 and 2012. Perhaps these ones might have had sex within marriage or consistently used condoms. Astonishingly, female respondents who engaged in non-marital sex in the last 12 months witnessed a decline in HIV prevalence. Possibly, they practised safer sex.

While HIV prevalence increased (although not significantly) among respondents who had their first sexual experience before age 15 years, it significantly declined among those who experienced sexual activity at age 15 years or more (not significant for males).

A further descriptive analysis was prompted by the findings in Table 1 on possible association of HIV prevalence and marital status vis-à-vis change in HIV prevalence between 2007 and 2012.

Table 2 presents a further exploratory descriptive analysis of HIV prevalence based on marital status. HIV prevalence was significantly associated with marital status both among males and females in both years of the survey. Across all the selected characteristics of interest, HIV prevalence was significantly higher for female respondents who were formerly married than either those currently married or never married for both 2007 and 2012. HIV prevalence for male respondents in the age group 15-19 years who were currently married is about three times higher than that of their female counterparts. However, the prevalence for formerly married female respondents in the same age group is 9.1 compared with no one positive among formerly married males. Urban males who were formerly married have higher prevalence compared with their counterparts in rural areas, and similar differences were noted among the females. Again, geopolitical zones are associated with marital status with respect to their HIV prevalence, especially for female respondents. Almost 15\% prevalence was found among formerly married women in the North East compared 3\% in the South East. HIV prevalence increases with wealth quintiles. HIV prevalence vis-à-vis sexual behaviour and practices of respondents were also significantly associated with their marital status. The HIV prevalence was generally higher among those involved in transactional sex and multiple sex partnering than those who were not, except among formerly married males ( 3.53 vs. 0.82 ). Also, across all marital statuses, prevalence was higher among those who experienced STIs recently.

Table 3 shows the values of the deviance information criterion used in deciding on the model fits. Apparently, model $M_{3}$ that contains the total (un-split) spatial effect, nonlinear effect of age and all the categorical variables except the knowledge indicator has the minimum values, thus proving the best fit for all the data sets. But a close look at the values reveals that the fits of $M_{3}$ are a slight improvement over $\mathrm{M}_{4}$, which includes the knowledge variable, while model $M_{1}$ provides the worst fit. Model $M_{4}$ is of particular interest in our results, because it contains all the variables considered.

Table 4 presents the estimates for the fixed effect parameters based on the posterior odds ratio and $95 \%$ credible intervals from logit models. The findings show that there was a significant decline in HIV between 2007 and 2012 for the combined data, but this was not significant for the male data when considered separately. Similarly, based on educational level, the results show that HIV prevalence was higher among respondents who attained a primary or secondary educational level compared with those with no education, but this was not significant in any of the split data sets. Based on geopolitical zones, the results show lower odds of HIV among respondents in the South East zone compared with those residing in the North Central zone, and this was significant for the combined, male and female data. Results based on the wealth index reveal that compared with respondents from the poorest households, HIV prevalence was high among those from the average, richer and richest households, and this was significant in the combined and split data sets. Currently married respondents have higher odds of contracting HIV compared with those not married, but this was not significant when the respondents were considered based on their gender. Among all the respondents, those who initiated sexual activities before age 15 years have a significantly higher likelihood of contracting HIV than those who had not had sex at the time of the survey. The results also show that male respondents have lower odds of contracting HIV compared with their female counterparts. 


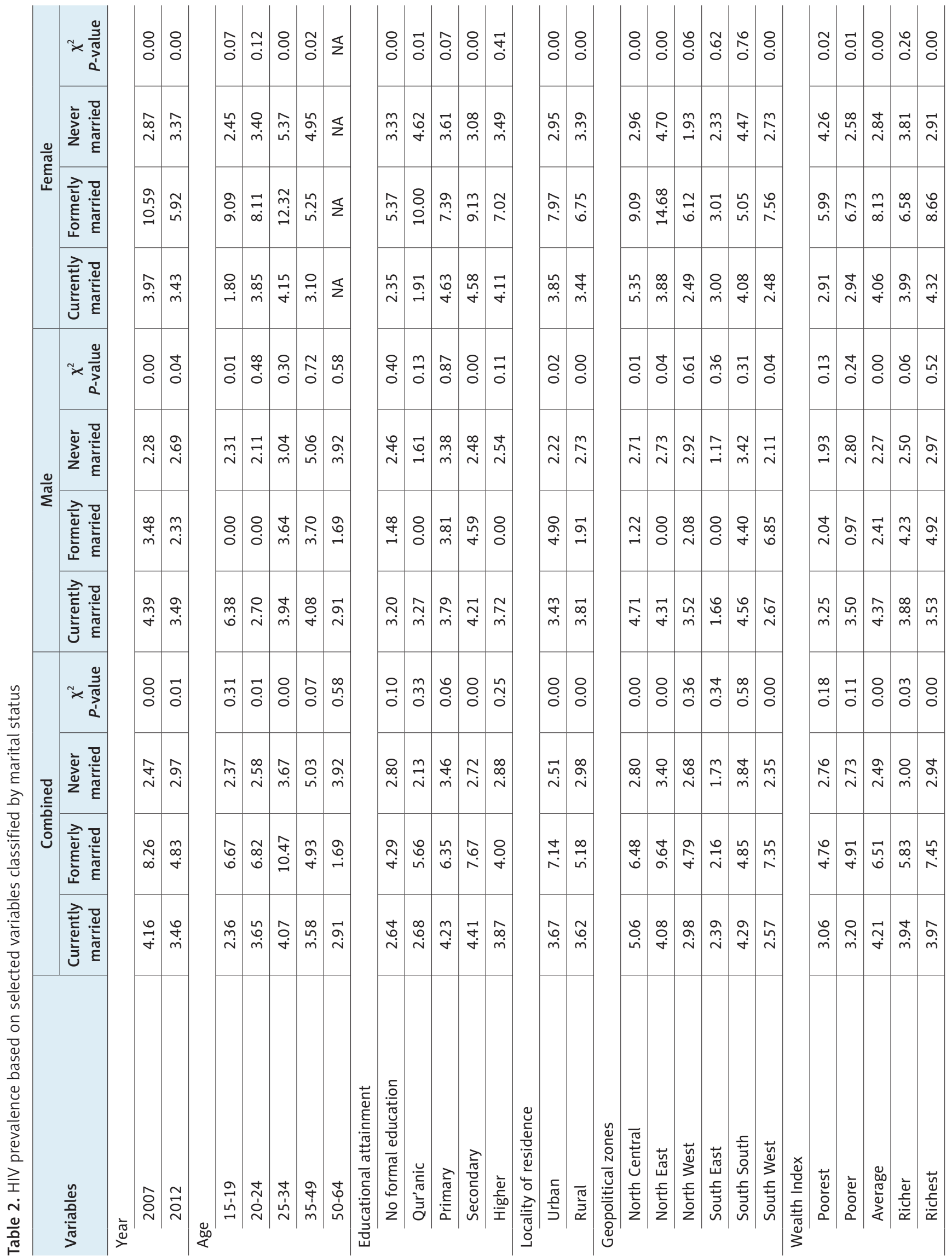




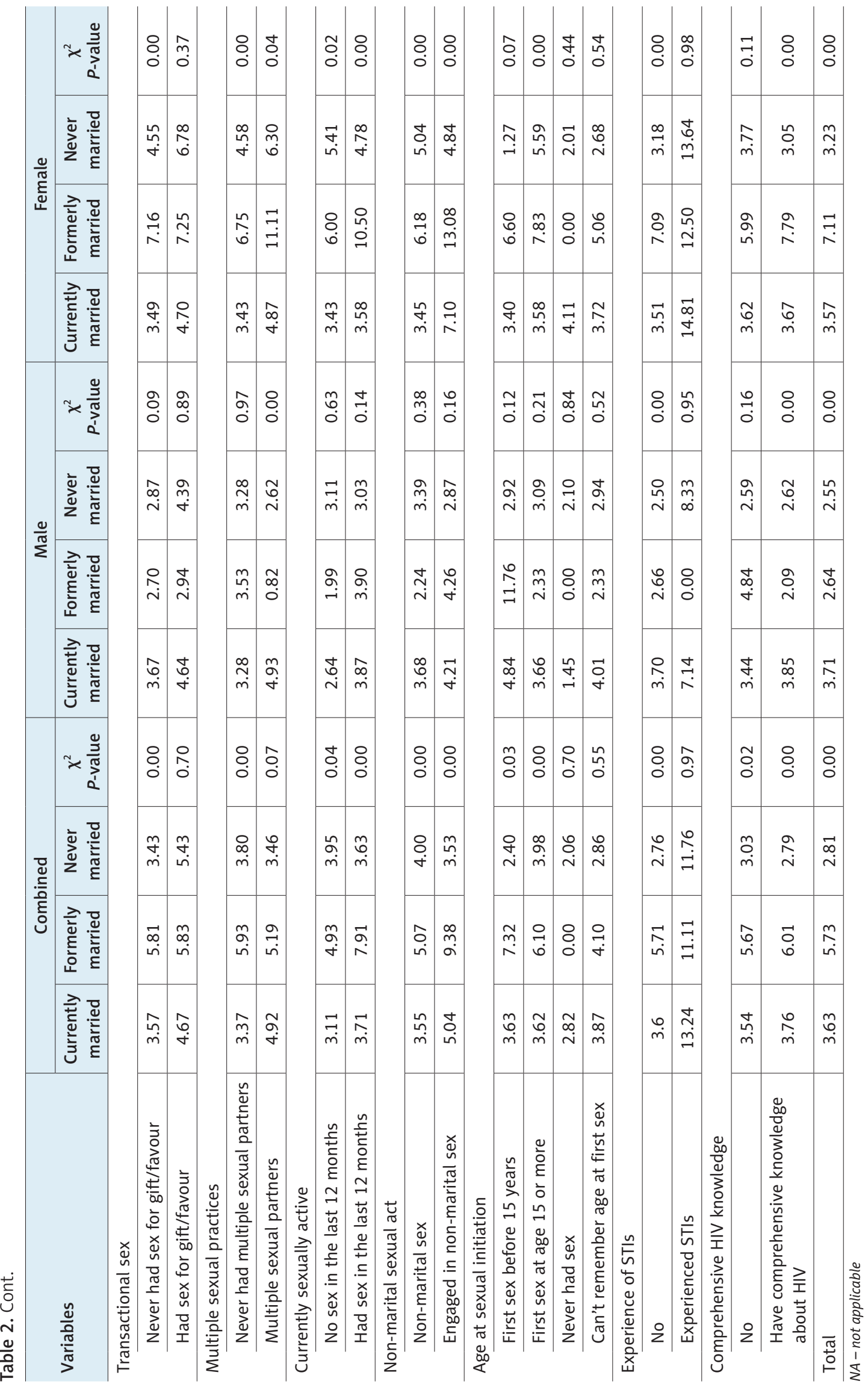


Table 3. Values of model diagnostic criteria, DIC

\begin{tabular}{l|c|c|c}
\hline Model & Female & Male & Combined \\
\hline$M_{1}$ & 4491.450 & 4491.702 & 9421.422 \\
\hline$M_{2}$ & 4427.122 & 4427.950 & 9229.474 \\
\hline$M_{3}$ & 3726.026 & 3725.495 & 8031.003 \\
\hline$M_{4}$ & 3727.541 & 3727.583 & 8033.177 \\
\hline$M_{5}$ & 4476.803 & 3911.901 & 8354.713 \\
\hline$M_{6}$ & 4293.478 & 3725.519 & 8033.145 \\
\hline
\end{tabular}

The nonlinear effects of respondents' age are presented in Figure 1A-C for all, male and female respondents. The results show a similar pattern for all the three datasets, implying that HIV prevalence is peak among middle-age individuals (around age 30-40 years), from where it declines with age.

The results of the spatial effects are presented in Figure 2A-F for all, male and female respondents. The figure shows the maps of the spatial effects (left panel) and those of credible intervals (right panel) used in deciding the significance of the estimated spatial effects. The states shaded in white in

Table 4. Posterior means and $95 \%$ credible intervals for the three models considered for female respondents

\begin{tabular}{|c|c|c|c|c|c|c|c|c|c|}
\hline \multirow{3}{*}{ Variables } & \multicolumn{3}{|c|}{ Combined } & \multicolumn{3}{|c|}{ Male } & \multicolumn{3}{|c|}{ Female } \\
\hline & \multirow{2}{*}{$\begin{array}{l}\text { Posterior } \\
\text { mean }\end{array}$} & \multicolumn{2}{|c|}{$95 \%$ Cr. interval } & \multirow{2}{*}{$\begin{array}{l}\text { Posterior } \\
\text { mean }\end{array}$} & \multicolumn{2}{|c|}{$95 \%$ Cr. interval } & \multirow{2}{*}{$\begin{array}{l}\text { Posterior } \\
\text { mean }\end{array}$} & \multicolumn{2}{|c|}{ 95\% Cr. interval } \\
\hline & & Lower & Upper & & Lower & Upper & & Lower & Upper \\
\hline Year of study & 0.827 & 0.721 & 0.949 & 0.840 & 0.671 & 1.052 & 0.836 & 0.679 & 1.048 \\
\hline Qur'anic & 1.046 & 0.754 & 1.428 & 0.972 & 0.637 & 1.359 & 0.951 & 0.627 & 1.490 \\
\hline Primary & 1.536 & 1.244 & 1.935 & 0.972 & 0.690 & 1.359 & 0.959 & 0.702 & 1.355 \\
\hline Secondary & 1.447 & 1.167 & 1.804 & 0.919 & 0.671 & 1.288 & 0.913 & 0.657 & 1.269 \\
\hline Higher & 1.037 & 0.786 & 1.381 & 0.676 & 0.439 & 1.026 & 0.667 & 0.458 & 1.006 \\
\hline Urban & 1.027 & 0.858 & 1.229 & 0.926 & 0.704 & 1.210 & 0.925 & 0.710 & 1.187 \\
\hline North West & 1.227 & 0.431 & 3.733 & 1.609 & 0.461 & 5.258 & 1.942 & 0.605 & 6.621 \\
\hline North East & 1.151 & 0.353 & 3.503 & 1.310 & 0.411 & 4.337 & 1.484 & 0.368 & 5.241 \\
\hline South West & 1.038 & 0.317 & 3.249 & 1.301 & 0.358 & 4.808 & 1.347 & 0.368 & 5.241 \\
\hline South South & 0.530 & 0.180 & 1.563 & 0.776 & 0.220 & 2.847 & 0.708 & 0.199 & 3.143 \\
\hline South East & 0.256 & 0.093 & 0.747 & 0.216 & 0.062 & 0.833 & 0.208 & 0.055 & 0.872 \\
\hline Poorer & 1.015 & 0.825 & 1.251 & 1.117 & 0.821 & 1.531 & 1.132 & 0.826 & 1.558 \\
\hline Average & 1.424 & 1.163 & 1.754 & 1.540 & 1.122 & 2.143 & 1.557 & 1.124 & 2.115 \\
\hline Richer & 1.433 & 1.120 & 1.186 & 1.563 & 1.104 & 2.236 & 1.583 & 1.126 & 2.235 \\
\hline Richest & 1.489 & 1.128 & 1.970 & 1.702 & 1.142 & 2.577 & 1.711 & 1.141 & 2.562 \\
\hline Currently married & 1.874 & 1.346 & 2.260 & 0.993 & 0.495 & 1.939 & 0.990 & 0.472 & 1.925 \\
\hline Formerly married & 1.170 & 0.920 & 1.501 & 1.216 & 0.849 & 1.735 & 1.228 & 0.863 & 1.734 \\
\hline Transactional sex & 1.114 & 0.890 & 1.365 & 1.162 & 0.820 & 1.582 & 1.151 & 0.819 & 1.567 \\
\hline Had multiple sexual partners & 1.161 & 0.981 & 1.391 & 1.199 & 0.972 & 1.476 & 1.202 & 0.975 & 1.495 \\
\hline Current sexual activities & 0.945 & 0.762 & 1.167 & 1.148 & 0.824 & 1.644 & 1.137 & 0.811 & 1.633 \\
\hline Non-marital sexual practices & 1.081 & 0.849 & 1.361 & 0.766 & 0.569 & 1.038 & 0.772 & 0.561 & 1.060 \\
\hline Sexual debut at $<15$ years & 5.999 & 2.496 & 27.956 & 0.00 & 0.000 & 0.001 & 0.568 & 0.000 & 266.459 \\
\hline $\begin{array}{l}\text { Can't remember age at } \\
\text { sexual debut }\end{array}$ & 6.774 & 2.496 & 32.812 & 0.000 & 0.000 & 0.001 & 0.599 & 0.000 & 287.323 \\
\hline Sexual debut at $\geq 15$ years & 6.517 & 2.656 & 30.417 & 0.000 & 0.000 & 0.001 & 0.471 & 0.000 & 224.589 \\
\hline $\begin{array}{l}\text { Experienced STIs in the last } \\
3 \text { months }\end{array}$ & 1.001 & 0.999 & 1.002 & 1.002 & 1.000 & 1.003 & 1.002 & 1.000 & 1.003 \\
\hline $\begin{array}{l}\text { Comprehensive knowledge } \\
\text { of HIV }\end{array}$ & 1.000 & 0.999 & 1.000 & 1.000 & 0.999 & 1.000 & 1.000 & 1.000 & 1.000 \\
\hline Male & 0.829 & 0.713 & 0.958 & NA & NA & NA & NA & NA & NA \\
\hline
\end{tabular}

NA - not applicable 
A

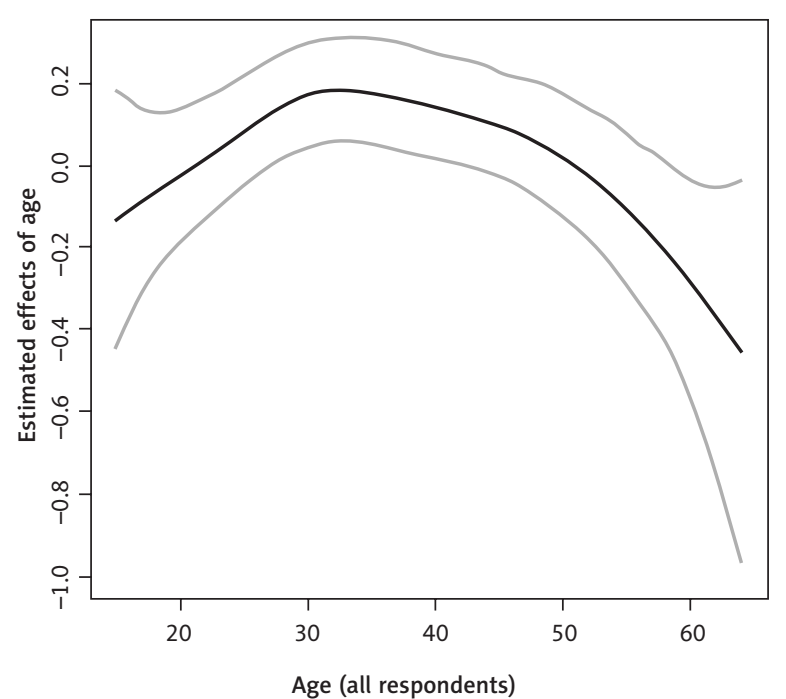

B

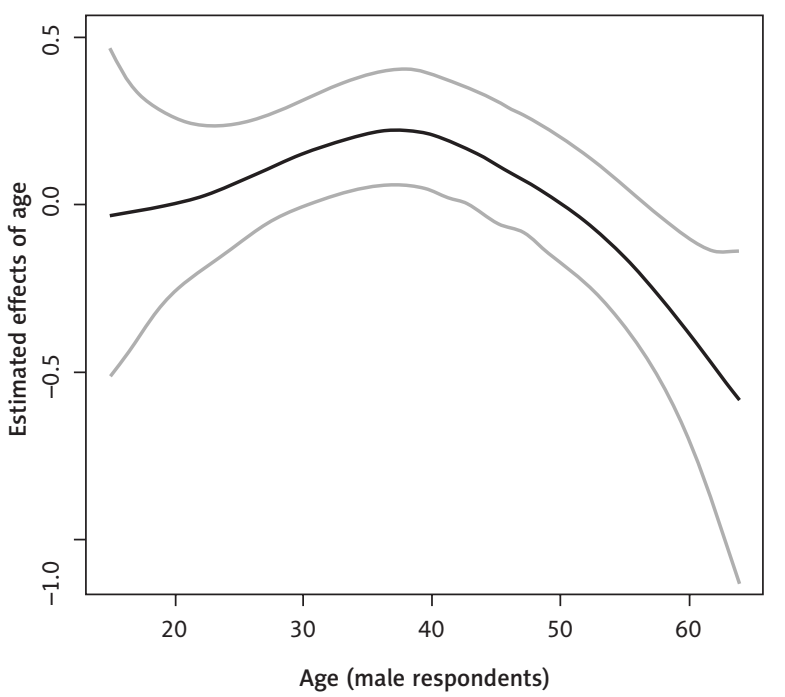

C

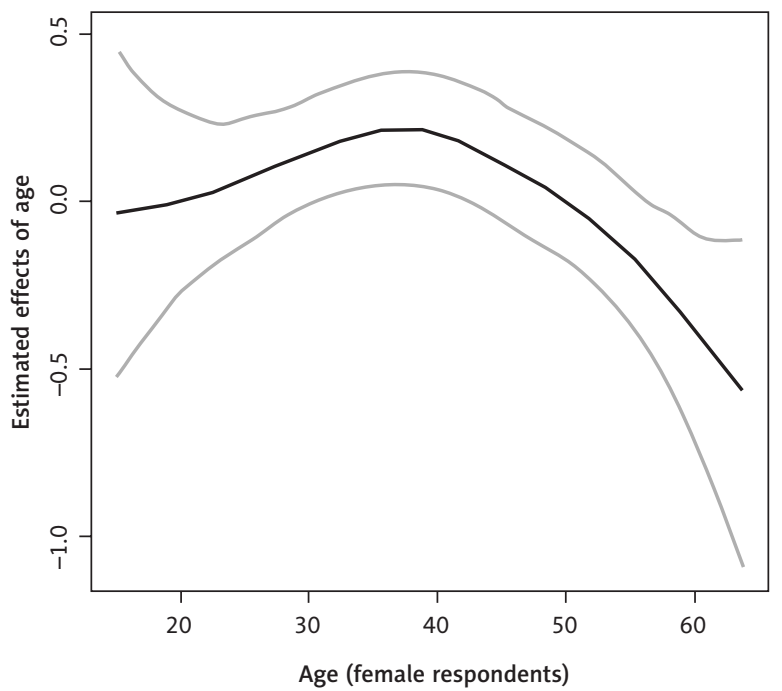

Figure 1. Nonlinear effect of age for A) all respondents, B) male respondents and C) female respondents. Included are the $95 \%$ credible intervals (in grey)

the left panel maps are those with significantly higher prevalence of HIV, those in black are those with a significantly lower prevalence, while estimates for states shaded in grey are not significant. The results show a similar pattern of significance for the three data sets. Specifically, for all respondents, HIV prevalence is significantly higher in neighbouring Benue Nasarawa and Abuja as well as in neighbouring Imo, Rivers and Akwa Ibom states, but significantly lower in Ekiti, Kebbi, and Zamfara states. For the male respondents, the findings show significantly higher prevalence in Benue, Nasarawa, Taraba, Rivers and Akwa Ibom states but lower in only Zamfara state, while for the female respondents, higher prevalences are recorded in Abuja, Benue, Nasarawa, Rivers and Akwa Ibom states but lower in Kebbi and Zamfara states.

\section{Discussion}

This study was performed to explore possible geographical variations in HIV prevalence among the general male and female population based on two waves of the National HIV/AIDS and Reproductive Health Surveys. We found significant spatial variations at a highly disaggregated level of states in Nigeria. The nonlinear effects of respondents' age show a similar pattern of HIV prevalence for male, female and the combined respondents, implying that HIV prevalence is peak among middle-aged individuals, from where it declines with age. Also, the results reveal a downward change in HIV prevalence in Nigeria between 2007 and 2012.

HIV prevalence was generally lower among the currently married respondents than the formerly married respon- 
A
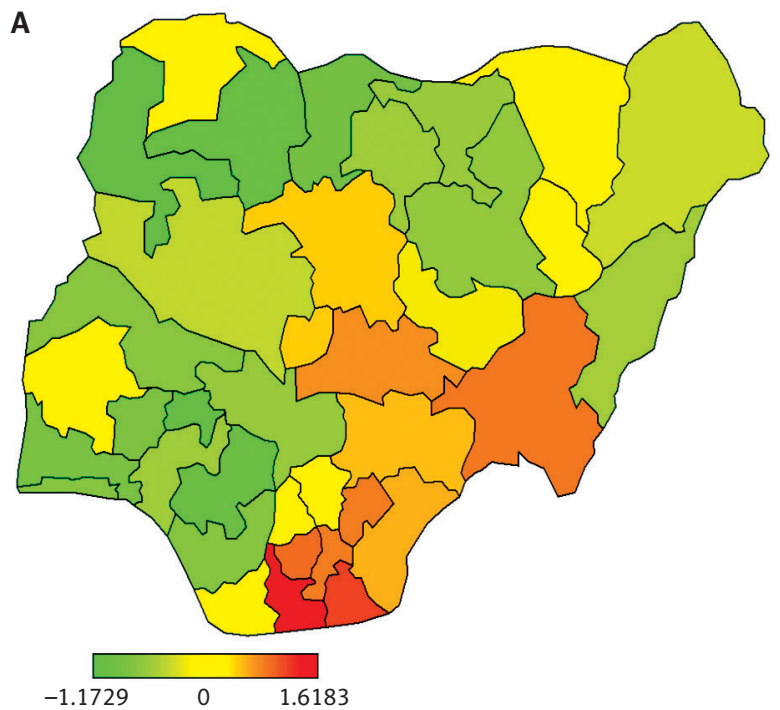

C
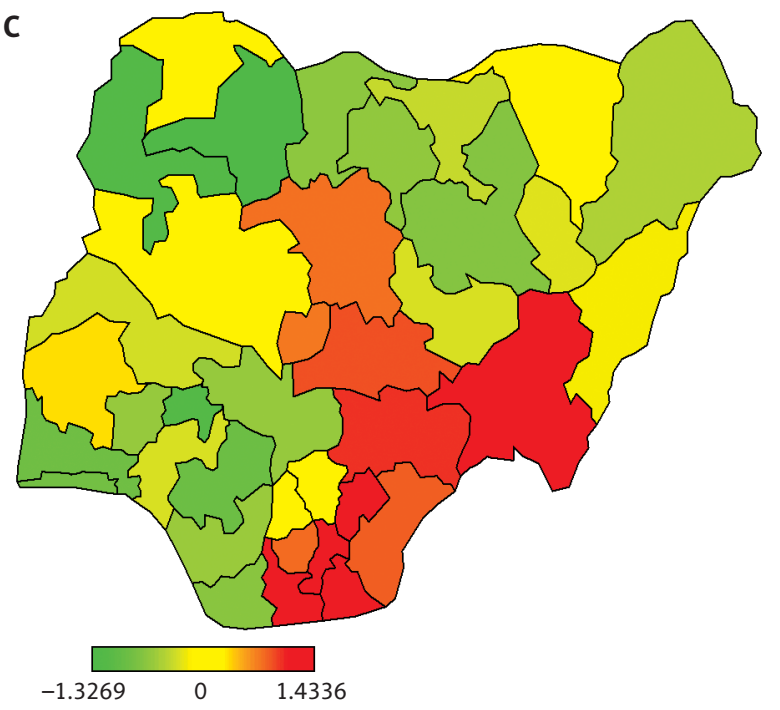

E
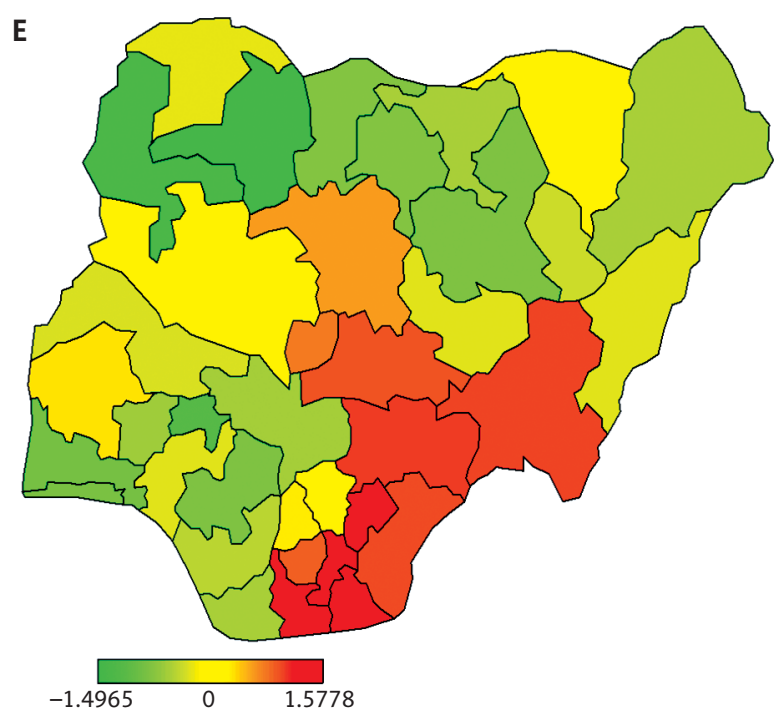

B

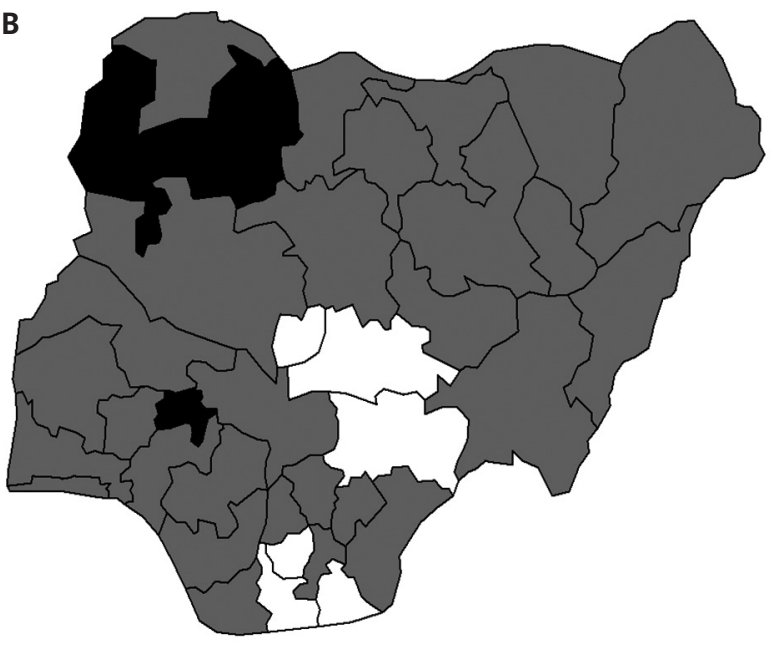

$\begin{array}{lll}-1 & 0 & 1\end{array}$

D

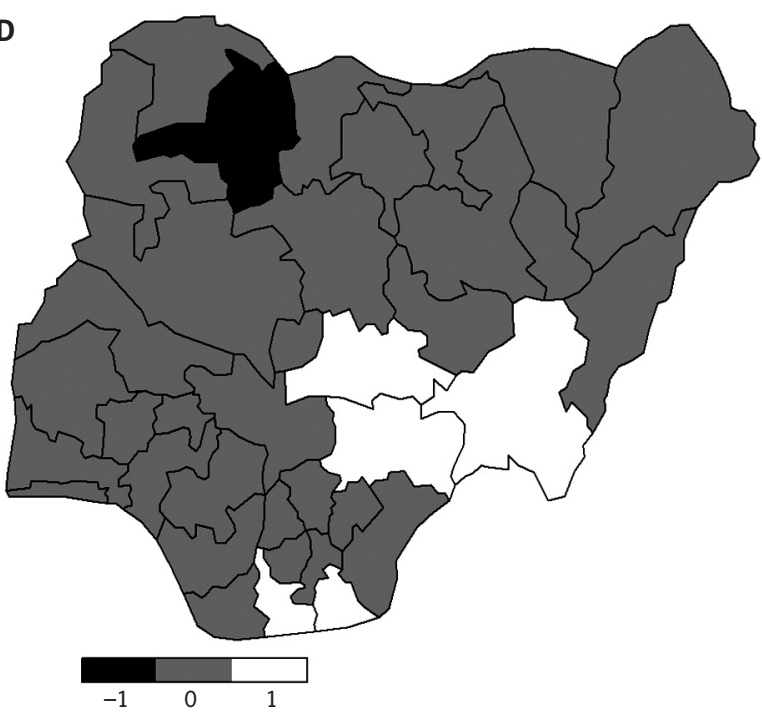

$\mathbf{F}$

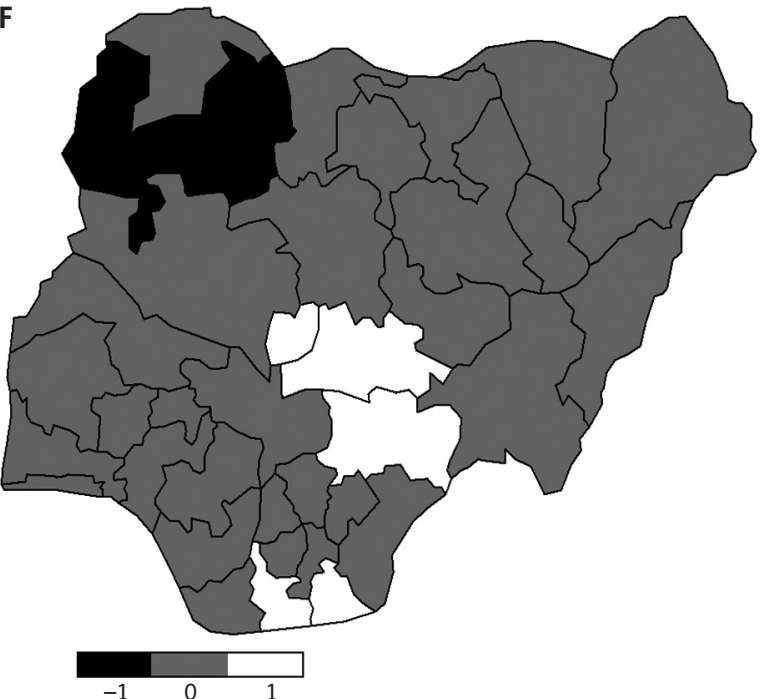

Figure 2. Maps of Nigeria showing the spatial effects for A) all respondents and B) the corresponding map of significance, C) for male respondents and $\mathbf{D}$ ) the corresponding map of significance, E) female respondents and $\mathrm{F}$ ) the corresponding map of significance 
dents, but least among never-married respondents. Similar outcomes have been reported in previous studies [33, 34]. Whereas it could be very alarming to note the decline from $10.59 \%$ in 2007 to $5.92 \%$ in 2012 (about 50\% reduction) in the prevalence among formerly married women, it is worrisome to see the increase in HIV prevalence from 2007 to 2012 among the single or never-married respondents (combined, male and female). Although this was not significant, it however calls for action to mitigate against a further rise in HIV prevalence in Nigeria.

We found significant spatial variations in HIV prevalence in Nigeria between 2007 and 2012 at the zonal levels. Generally, there is a decline in HIV prevalence for female (significant for North Central and South West), but a significant increase was noted for males in the North East. Similar geographical variations have been reported earlier [35].

Shockingly, HIV prevalence increased between 2007 and 2012 among the respondents who said they had never had sex. This leaves many questions to be answered and indeed mysteries to be unravelled! Is it that the respondents did not answer the questions sincerely? Could they have contracted it through other, non-sexual modes of transmission? Or could it be due to false disclosure of sexual acts? Although there are other ways of HIV transmission besides sexual intercourse, sexual transmission is the main channel of HIV transmission [14, 15, 20]. This worrisome finding, therefore, gives room for the possibility that the "never had sex" respondents might have been infected through other channels, such as mother-to-child transmission, injections, sharing of sharp objects, or blood transfusion. Nevertheless, the prevalence of $1.6 \%$ in 2007 and $2.3 \%$ in 2012 found among the "never had sex" population is generally low compared with $6.9 \%$ found among virgins in a South African study, although the prevalence among the sexually active people in the study was as high as $15.9 \%$ [33].

Our finding that an association existed between HIV prevalence and marital status vis-à-vis change in HIV prevalence between 2007 and 2012 is worthy of note. With formerly married women (divorced, separated or widowed) having the highest HIV prevalence, it is important to explore this further to understand major confounders of HIV prevalence and marital status. A similar study $[19,20]$ corroborated this but with a less flexible approach that can permit spatial estimation at highly disaggregated state levels.

HIV prevalence was significantly higher for female respondents who were formerly married than either those currently married or never married for both 2007 and 2012. Similar variations have been documented $[20,36]$. The zero prevalence found among formerly married males across some characteristics could be ascribed to the low sample size in that category. Ordinarily, most males are never married at age 15 , making it uncommon to come across males aged 15-19 years who were already formerly married. Educational attainment is significantly associated with marital status with respect to HIV prevalence among female respondents up to secondary education.
Our findings on the effects of respondents' age revealed that a similar pattern exists for both males and females with HIV prevalence peaking at middle age. This might be expected since studies have shown that in most sub-Saharan African countries, fertility, which is a product of sexuality, is peak among this age group [37].

\section{Conclusions}

This study was motivated by the need to disentangle the spatial distribution of HIV prevalence in Nigeria. This is particularly important considering the renewed efforts by the government to combat the spread of the infectious disease in Nigeria. The descriptive aspect of the study has revealed a downward trend in HIV prevalence in Nigeria between 2007 and 2012 and this was found to be significant in the case of all the respondents in the Bayesian spatial model. Some sub-Saharan African countries with high prevalence of HIV have equally been reported to have recorded downward trends in recent years, a situation attributed to improved knowledge as a result of aggressive campaigns by government and non-governmental agencies in addition to increased use of contraceptives $[38,39]$. More sustained and yet effective strategies that could avert increased incidence especially among the most-at-risk individuals in the country still need to be put in place.

Like findings from other studies [40], the study has also found the prevalence of HIV higher among women than their male counterparts. The patriarchal system in Nigeria ensures weaker women's rights such that they have little or no control over issues pertaining to their health, including the use of contraceptives. In this setting, they are more exposed to the risks of HIV even if they are married to one husband, who may have more than one wife or may be having extramarital sexual activities. An effective intervention strategy should, therefore, include efforts to ensure that women are empowered irrespective of their marital status, to protect themselves against risky behaviours that could increase their probability of contracting HIV or any other infectious diseases.

The method of spatial analysis allows for segregating the prevalence by states such that one can determine locations with significantly higher or lower prevalence. The prevalence of HIV varies considerably according to the respondents' geographical locations. This provides insight into the effective utilization of the scarce resources which can be achieved through cost-effective HIV prevention strategies by proper prioritization of needs. State-specific interventions that could achieve a downward trend in states with higher prevalence need to be adopted. This could include some cultural and behavioural changes suitable to those locations and that can be easily accepted by the locals. On the other hand, intervention strategies that could ensure behavioural maintenance need to be adopted so that the successes attained in those locations would not be eroded with time. 


\section{Conflict of interest}

The authors declare no potential conflicts of interest with respect to the research, authorship, and/or publication of this article.

\section{References}

1. Manda SO, Lombard CJ, Mosala T. Divergent spatial patterns in the prevalence of the human immunodeficiency virus (HIV) and syphilis in South African pregnant women. Geospatial Health 2012; 6: 221-231.

2. Saphonn V, Hor LB, Ly SP, Chhuon S, Saidel T, Detels R. How well do antenatal clinic (ANC) attendees represent the general population? A comparison of HIV prevalence from ANC sentinel surveillance sites with a population-based survey of women aged 15-49 in Cambodia. Int J Epidemiol 2002; 31: 449-455.

3. World Health Organization, Joint United Nations Programme on HIV/AIDS. Reconciling antenatal clinic-based surveillance and population-based survey estimates of HIV prevalence in sub Saharan Africa. WHO and UNAIDS, Geneva 2003.

4. Zaba B, Carpenter LM, Boerma JT, Gregson S, Nakiyingi J, Urassa M. Adjusting ante-natal clinic data for improved estimates of HIV prevalence among women in sub-Saharan Africa. AIDS 2000; 14: 27412750 .

5. MacQueen K. The Epidemiology of HIV Transmission: Trends, Structure and Dynamics. Ann Rev Anthropol 1994; 23: 509-526.

6. UNAIDS. Global HIV \& AIDS statistics - 2018 fact sheet, 2018. Available at: http://www.unaids.org/en/resources/fact-sheet.

7. WHO. HIV/AIDS Data and Statistics, 2018. Available at: http:// www.who.int/hiv/data/en/.

8. WHO. Global Health Observatory (GHO) Data. Geneva, 2015. Available at: http://www.who.int/gho/hiv/en/.

9. UNAIDS. Prevention Gap Report. Washington, 2016. Available at: http://www.unaids.org/sites/default/files/media_asset/2016-prevention-gap-report_en.pdf.

10. ActionAid. Poverty and HIV/AIDS, 2003. Available at: http://www. actionaid.org/ourpriorities/hiv/paha/paha.shtml.

11. Craddock S. Beyond epidemiology: locating AIDS in Africa. In: Kalipeni E, Craddock S, Oppong JR, Ghosh J (eds.). HIV and AIDS in Africa: Beyond Epidemiology. Wiley-Blackwell, 2003.

12. FMOH. National HIV/AIDS and Reproductive Health and Serological Survey, 2012 (NARHS Plus). Federal Ministry of Health, Abuja 2013.

13. NPC. Nigeria over 167 million. National Population Commission. Nigeria, 2013; Available at: http://www.population.gov.ng/index. php/84-news/latest/106-nigeria-over-167-million-population-implications-and-challenges.

14. NACA. Global AIDS Response Country Progress Report - Nigeria GARPR. National Agency for the Control of AIDS, Abuja 2015.

15. FMOH. National HIV/AIDS and Reproductive Health and Serological Survey, 2012 (NARHS Plus). Federal Ministy of Health, Abuja 2013.

16. FMOH. National HIV sero-prevalence sentinel survey among pregnant women attaending antenatal clinics in Nigeria. Federal Ministry of Health, Abuja 2010.

17. FMOH. Integrated Biological and Behavioural Surveillance Survey (IBBSS). Federal Ministry of Health, Abuja 2010.

18. FMOH. National HIV/AIDS and Reproductive Health and Serological Survey, 2007 (NARHS Plus). Federal Ministry of Health, Abuja 2008.

19. Adebayo SB, Olukolade RB, Idogho O, et al. Marital status and HIV prevalence in Nigeria: implications for effective prevention programmes for women. Adv Infect Dis 2013; 3: 210-218.

20. Fagbamigbe FA, Adebayo SB, Idemudia E. Marital status and HIV prevalence among women in Nigeria: ingredients for evidence-based programming. Int J Infect Dis 2016; 48: 57-63.
21. Gayawan E, Arogundade ED, Adebayo SB. A Bayesian multinomial modeling of spatial pattern of co-morbidity of malaria and nonmalarial febrile illness among young children in Nigeria. Trans $\mathrm{R}$ Soc Trop Med Hyg 2014; 108: 415-424.

22. Gayawan E, Arogundade ED, Adebayo SB. Possible determinants and spatial patterns of anaemia among young children in Nigeria: a Bayesian semi-parametric modelling. Int Health 2014; 6: 35-45.

23. Johnson K, Way A. Risk factors for HIV infection in a national adult population: evidence from the 2003 Kenya Demographic and Health Survey. J Acquir Immune Def Syndr 2006; 42: 627-636.

24. Ngesa O, Mwamb H, Achia T. Bayesian spatial semi-parametric modeling of HIV variation in Kenya. PLoS One 2014; 9: e103299.

25. Eze JI. Modelling HIV/AIDS Epidemic in Nigeria. University of Glasgow, 2009.

26. Mishra V, Vaessen M, Boerma JT, et al. HIV testing in national population-based surveys: experience from the Demographic and Health Surveys. Bull World Health Organ 2006; 84: 537-545.

27. Hastie T, Tibshirani R. Generalized additive models. Chapman and Hall, London 1990.

28. Lang S, Brezger A. Bayesian P-splines. J Comput Graph Stat 2004; 13: $183-212$.

29. Brezger A, Lang S. Generalized structured additive regression based on Bayesian P-splines. Computational Statistics and Data Analysis 2006; 50: 967-991.

30. Rue H, Held L. Gaussian Markov random fields. Theory and applications. Chapman \& Hall/CRC, Boca Raton 2005.

31. Besag J, York Y, Mollie A. Bayesian image restoration with two applications in spatial statistics (with discussion). Annals of the Institute of Statistical Mathematics 1991; 43: 1-59.

32. Spiegelhalter DJ, Best NG, Carlin BP, Van der Linde A. Bayesian measures of model complexity and fit. J R Statist Soc B 2002; 64: 583-639.

33. Shisana O, Toefy Y, Simbayi LC, Simbayi LC, Malik S, Zuma K. Marital status and HIV infection in South Africa. S Afr Med J 2004; 94 : 537-543.

34. Maan MA, Hussain F, Jamil M. Prevalence and risk factors of HIV in Faisalabad, Pakistan - a retrospective study. Pakistan Journal of Medical Science 2014; 30: 32-35.

35. Slaymaker E, Zaba B. Sexual behaviour change, marriage and HIV prevalence in Zambia. In: Carael C, Zaba B (eds.). HIV, resurgent infectiouns and population change in Africa. Springer, 2007; 155-170.

36. Kposowa AJ. Marital status and HIV/AIDS mortality: evidence from the US National Longitudinal Mortality Study. Int J Infect Dis 2013; 17: e868-e874.

37. Gayawan E, Adebayo SB, Ipinyomi RA, Oyejola BA. Modeling ferility curves in Africa. Demographic Research 2010; 22: 211-236.

38. UNAIDS. Report on the Global AIDS Epidemic, 2008.

39. Adeyemo BO, Gayawan E, Olusile AO, Komolafe IOO. Prevalence of HIV infection among pregnant women presenting to two hospitals in Ogun state, Nigeria. HIV AIDS Rev 2014; 13: 90-94.

40. Awofala AA, Ogundele OE. HIV epidemiology in Nigeria. Saudi Journal of Biological Sciences 2018; 25: 697-703. 